\title{
Indicadores
}

\section{Indicadores de uso y participación en las revistas científicas 2.0: el caso de PLoS One}

\author{
Por Álvaro Cabezas-Clavijo y Daniel Torres-Salinas
}

Resumen: Los nuevos entornos de publicación y comunicación científica han propiciado la aparición de nuevos indicadores Web. Junto a las métricas de uso, como las descargas, existen múltiples medidas que se generan a partir de la web 2.0 científica. Las revistas de la Public Library of Science recopilan de forma sistemática gran parte de estas nuevas métricas. Se presentan algunos de estos indicadores y su análisis cuantitativo mediante un estudio de caso a partir de 8.945 artículos publicados en la revista PLoS One. Los indicadores seleccionados han sido: número de comentarios, puntuación, número de bookmarks, enlaces desde blogs científicos, número de descargas, número de vistas y número de citas. Para todos ellos se han calculado sus estadísticas básicas así como las correlaciones entre ellas. Los resultados revelan la escasa participación de los científicos en la web 2.0 y cómo la mayor parte de estos indicadores, exceptuando las descargas y visitas, son medidas poco consolidadas.

Palabras clave: Ciencia 2.0, Web 2.0, PLoS One, Comunicación científica, Indicadores, Revistas.

Title: Indicators for usage and participation in scientific journals 2.0: the case of PLoS One.

Abstract: The new publishing and scientific communication environments have led to the emergence of new Web indicators. Along with usage metrics such as downloads there are many measures that are generated from Science 2.0. Journals

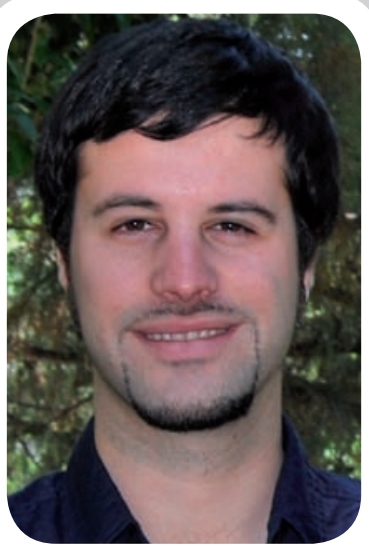

Álvaro Cabezas-Clavijo, licenciado en documentación y máster en Información Científica por la Univ. de Granada, es miembro del grupo EC3 de la misma universidad, donde se dedica al estudio de la actividad científica desde una perspectiva bibliométrica. Sus líneas de investigación son la evaluación cienciométrica de agentes investigadores así como el impacto de las herramientas de la web 2.0 en la actividad investigadora.

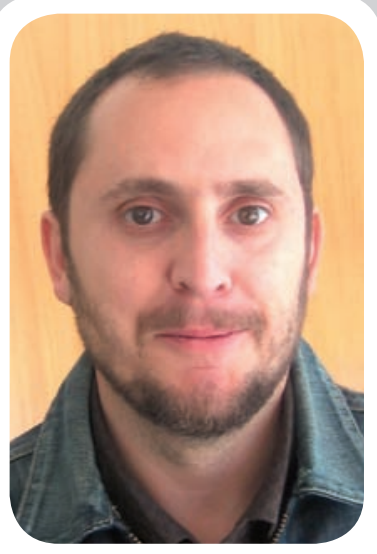

Daniel Torres-Salinas es doctor en documentación y trabaja como técnico de gestión de la investigación en la Universidad de Navarra, donde evalúa la calidad y el impacto de la investigación mediante de indicadores bibliométricos. Asimismo es miembro del grupo EC3 (Evaluación de la Ciencia y de la Comunicación Científica) de la Universidad de Granada donde participa en diferentes contratos y proyectos.

published by the Public Library of Science systematically collect many of these new metrics. The objective of this paper is to present some of these new indicators and analyze them quantitatively through the case study of 8945 papers published in the journal PLoS One. The selected indicators were; comments, ratings, number of bookmarks, links from scientific weblogs, downloads views and citations. Basic descriptive statistics indicators and correlations have been calculated for all of them. The results show the low participation of scientists in Web 2.0 and how most of these indicators, except for downloads and visits, are poorly consolidated metrics.

Keywords: Science 2.0, Web 2.0, PLoS One, Scientific communication, Indicators, Journals.

Cabezas-Clavijo, Álvaro; Torres-Salinas, Daniel. "Indicadores de uso y participación en las revistas científicas 2.0: el caso de PLoS One". El profesional de la información, 2010, julio-agosto, v. 19, n. 4, pp. 431-434.

DOI: $10.3145 /$ epi.2010.jul.14

\section{Introducción}

\section{LA FRAGMENTACIÓN DE} LA COMUNICACIÓN CIENTÍFICA con la reciente llegada al mercado de la publicación académica de nuevos actores (repositorios y editoriales de acceso abier- to) abre diversas interrogantes en el ámbito de la información científica, así como en la evaluación de su impacto (Torres-Salinas; Delgado-López-Cózar, 2009).

Aunque la bibliometría cuenta con una amplia gama de indicado- res basados en el número de citas para valorar la repercusión de las publicaciones, no ofrece, hasta el momento, respuestas coherentes en la medición del uso de los materiales académicos online. Sin embargo las nuevas capacidades de la web permiten que en los reposito- 
rios estén implementando métricas destinadas a captar el uso de los materiales que recopilan. Los indicadores de uso se convierten de este modo en una nueva vía de medir la actividad científica, especialmente si, como indican algunos estudios, la disponibilidad de los mismos es uno de los principales factores que determina el uso de las publicaciones académicas (Evans, 2008).

Los proyectos Mesur (Metrics from scholarly usage of resources), Counter (Counting online usage of networked electronic resources) o el nacimiento de la denominada "usage bibliometrics" (Kurtz; Bollen, 2010) son hitos que muestran el interés del mundo académico por dotarse de nuevas aplicaciones para la valoración de sus actividades. Por otro lado la ciencia 2.0, conformada por múltiples plataformas (blogs, páginas de favoritos, gestores de referencias, etc.) en las que es posible la interacción de los usuarios en el mundo académico (Cabezas-Clavijo; Torres-Salinas; Delgado-LópezCózar, 2009) abre vías alternativas a la medición del uso y de la popularidad de los materiales. La posibilidad de medir cuántas veces se comparte un artículo con la red de contactos o cuántas veces se visualiza una presentación online, genera una serie de nuevos indicadores estrechamente relacionados con la web 2.0.

http://www.mesur.org/ http://www.projectcounter.org/

Uno de los sitios que mejor representa la apuesta por estas nuevas medidas es el de las revistas científicas en acceso abierto de la editorial Public Libray of Science (PLoS). Recientemente este conjunto de revistas implementaron para cada uno de los artículos que publicaban una serie de métricas para captar la repercusión, el uso y el impacto que éstos obtenían en la comunidad científica (Neylon; Wu, 2009). Estas medidas combinan indicadores tradicionales como el número de citas, con métricas de difusión en la web 2.0, así como con indicadores de participación y de uso. Todos ellos se recopilan para los trabajos publicados en las revistas científicas de la editorial $P L o S$ por lo que es una fuente de información única. El análisis de estas métricas nos sirve para plantear los objetivos de este trabajo:

1. Presentar algunas de las nuevas métricas de artículos (article level metrics) que PLoS utiliza para evaluar las distintas dimensiones de uso y difusión de sus artículos.

2. Determinar el uso por parte de los usuarios de las funciones 2.0 que la revista ofrece (comentarios, ratings, etc.).

3. Establecer relaciones de dependencia entre los diversos indicadores.

http://article-level-metrics.plos.org/

\section{"Los repositorios implementan métricas de uso de los materiales que recopilan"}

\section{Metodología}

Los datos se obtuvieron de la propia editorial Public Libray of Science (PLOS), que pone a disposición pública un archivo con los datos brutos de cada uno de los artículos publicados por sus siete revistas. Hemos seleccionado como caso de estudio la revista PLoS One que ha publicado desde finales de 2006 a comienzos de 2010 un total de 8.945 artículos. El análisis de los datos se basa en el fichero de $P L O S$ actualizado a 31 de enero de 2010 , que presenta un total de 23 indicadores diferentes. Para facilitar el análisis de los mismos se han seleccionado los más significativos, que hemos agrupado en cuatro dimensiones (figura 1).

http://www.plos.org/downloads/plosalm.zip

\section{Dimensión 1: Interacción y parti- cipación del usuario}

- Número de comentarios y notas. Los lectores pueden realizar comentarios en los propios artículos así como guardar notas en cualquier parte del texto. La revista pretende así fomentar la discusión de los contenidos de los artículos.

- Puntuaciones a los artículos. Los usuarios pueden puntuar los artículos en tres apartados en una escala de 1 a 5 , o simplemente valorar la calidad general del artículo.

\section{Dimensión 2: Uso de los artículos}

- Número de descargas pdf que los usuarios realizan desde la web de la revista. Se trata del indicador de uso más habitual. Los datos de PLoS siguen el estándar Counter.

- Número vistas/visitas o veces que se ha accedido al texto completo del artículo en la versión onlinehtml, sin descargar el pdf.

\section{Dimensión 3: Difusión en la web 2.0}

- Número de enlaces y menciones que cada artículo obtiene en los portales especializados de blogs científicos: Postgenomic, Nature blogs y ResearchBlogging.

- Número de bookmarks. Veces que cada artículo es marcado como favorito en los sitios de etiquetado social Connotea y Citeulike, ampliamente utilizados por la comunidad científica.

\section{Dimensión 4: Reconocimiento tradicional}

- Número de citas. Se recoge asimismo un indicador tradicional

\footnotetext{
"En el caso de PLoS One se constata la baja utilización de sus funciones 2.0 y la escasa difusión de sus trabajos en la web social"
} 


\begin{tabular}{|c|c|}
\hline $\begin{array}{c}\text { DIMENSIÓN } 1 \\
\text { Interacción y participación } \\
\text { - N de comentarios } \\
\text { - Puntuación (rating) }\end{array}$ & $\begin{array}{c}\text { DIMENSIÓN } 3 \\
\text { Difusión en la web } 2.0 \\
\text { - Enlaces desde blogs científicos } \\
\text { • } \mathbf{N}^{\circ} \text { de bookmarks }\end{array}$ \\
\hline $\begin{array}{c}\text { DIMENSIÓN } 2 \\
\text { Uso de los artículos } \\
\cdot N^{\circ} \text { de descargas } \\
\cdot N^{\circ} \text { de vistas/visitas }\end{array}$ & $\begin{array}{c}\text { DIMENSIÓN } 4 \\
\frac{\text { Reconocimiento tradicional }}{\cdot N^{\circ} \text { de citas en Scopus }}\end{array}$ \\
\hline $\begin{array}{l}\text { Average Rating } \\
\text { Rate This Article }\end{array}$ & $\begin{array}{l}\text { Bookmarked in } \\
\text { No related bookmarks found } \\
\text { Blog Coverage } \\
\text { Search for related blog posts }\end{array}$ \\
\hline $\begin{array}{l}\text { Article Usage (1) } \\
\text { Total Article Views: } \mathbf{8 9 2 4} \\
\text { Breakdown by View Type } \\
\text { HTML Page Views: } 8390 \\
\text { PDF Downloads: } 473 \\
\text { XML Downloads: } \mathbf{6 1}\end{array}$ & $\begin{array}{l}\text { Citations } \\
\text { Cited in } \\
\text { CrossRef (5), PubMed Central ( } 6 \text { ), Scopus ( } 5 \text { ) } \\
\text { Search for citations in Google Scholar. }\end{array}$ \\
\hline
\end{tabular}

Figura 1. Indicadores clasificados según su dimensión e implementación en la revista PLoS One.

de impacto científico, como son las citas. Para el cálculo de este indicador se usa la base de datos Scopus.

\section{Resultados}

La tabla 1 muestra el resumen de estadísticas para los distintos indicadores. Se ha incluido el porcentaje de artículos con valor 0 para cada una de las medidas propuestas. La dimensión 1 de interacción y participación muestra unos promedios bajos para los dos indicadores calculados, a la par que un altísimo porcentaje de artículos que no reci- (los más antiguos han dispuesto de más tiempo para ser descargados) promediando 421 descargas los artículos de 2006 y únicamente 38 los escasos artículos recogidos en 2010. El mismo efecto se produce en la contabilización de las visitas a los artículos. Respecto a la medición a partir de las citas recibidas, casi la mitad de los artículos no recibieron citas. Si excluimos de este análisis los años 2009 y 2010, que han gozado de poco tiempo para ser citados, el porcentaje de artículos no citados se sitúa en un $14 \%$. Por su parte, el promedio de citas está entre las 10 citas por artículo del año 2006 y las 4 de 2008.

La relación entre los indicadores la hemos calibrado con los coeficientes de correlación (tabla 2). La más elevada se produce entre el número de visitas a los artículos y las descargas efectuadas, con 0,63. Por su parte, la relación entre el número de citas en Scopus y el de descargas apenas llega a 0,52. El resto de pares de correlaciones alcanzan valores aún menores y por tanto parece no existir relación entre los indicadores. sión 2.0, con casi un $90 \%$ de artículos que no han sido enlazados desde los servidores de blogs científicos. Asimismo los valores máximos y totales de dichas dimensiones están lejos del número de descargas o del número de citas.

La dimensión 2, de uso de la investigación, presenta valores promedios en cuanto a descargas de 215 por artículo, si bien la fecha de publicación juega un papel relevante

\section{"Los bajos valores que se obtienen en comentarios, puntuación o difusión en blogs evidencian que no son indicadores consolidados"}

\begin{tabular}{|c|c|c|c|c|c|c|c|}
\hline & I ndicador & Total & Promedio & Mediana & Moda & Máximo & $\begin{array}{l}\% \text { artícs. } \\
\text { valor } 0\end{array}$ \\
\hline \multirow{2}{*}{$\begin{array}{l}\text { Dimensión } 1 \\
\text { Interacción }\end{array}$} & Comentarios/Notas & 3.547 & 0,4 & 0 & 0 & 48 & $82 \%$ \\
\hline & Puntuaciones & 997 & 0,1 & 0 & 0 & 22 & $91 \%$ \\
\hline \multirow{2}{*}{$\begin{array}{l}\text { Dimensión } 2 \\
\text { Uso }\end{array}$} & Descargas pdf & 1.924 .748 & 215,0 & 156 & 150 & 6.534 & $0 \%$ \\
\hline & Vistas/Visitas & 10.878 .304 & $1.216,0$ & 686 & 474 & 143.803 & $0 \%$ \\
\hline \multirow{2}{*}{$\begin{array}{l}\text { Dimensión } 3 \\
\text { Difusión } 2.0\end{array}$} & Enlaces blogs & 1.589 & 0,2 & 0 & 0 & 15 & $89 \%$ \\
\hline & Bookmarks & 4.172 & 0,5 & 0 & 0 & 47 & $77 \%$ \\
\hline $\begin{array}{l}\text { Dimensión } 4 \\
\text { Reconocimiento }\end{array}$ & Citas & 23.418 & 2,6 & 1 & 0 & 115 & $47 \%$ \\
\hline
\end{tabular}

Tabla 1. Valores de las medidas estadísticas descriptivas de los indicadores de PLoS One 


\begin{tabular}{|c|c|c|c|c|c|c|c|}
\hline & Citas & Visitas & Descargas & Enlaces & Bookmarks & Puntuaciones & $\begin{array}{c}\text { Comentarios/ } \\
\text { Notas }\end{array}$ \\
\hline Citas & 1,00 & -- & --- & -- & -- & -- & -- \\
\hline Visitas & 0,24 & 1,00 & -- & -- & -- & -- & -- \\
\hline Descargas & 0,52 & 0,63 & 1,00 & -- & -- & -- & --- \\
\hline Enlaces & 0,14 & 0,45 & 0,45 & 1,00 & -- & -- & -- \\
\hline Bookmarks & 0,14 & 0,18 & 0,32 & 0,16 & 1,00 & -- & -- \\
\hline Puntuaciones & 0,12 & 0,21 & 0,23 & 0,19 & 0,13 & 1,00 & -- \\
\hline $\begin{array}{l}\text { Comentarios/ } \\
\text { Notas }\end{array}$ & 0,21 & 0,31 & 0,35 & 0,30 & 0,13 & 0,43 & 1,00 \\
\hline
\end{tabular}

Tabla 2. Coeficientes de correlación entre los indicadores de PLoS One

\section{Consideraciones finales}

Este trabajo nos ha permitido presentar indicadores de uso y participación en revistas científicas online mediante el estudio de caso de PLoS One. Se constata la baja utilización de sus funciones $2.0 \mathrm{y}$ la escasa difusión de sus trabajos en la web social. Los bajos valores que se obtienen en indicadores como los comentarios, la puntuación o los relacionados con la difusión en blogs y sistemas de bookmarking evidencian que no son indicadores consolidados. Parece que no ofrecen demasiados datos complementarios frente a otros indicadores como las citas o las descargas, que sí presentan información útil y lo suficientemente amplia tanto al editor como al investigador. Así mismo, el hecho de que entre el $77 \%$ y el $91 \%$ de los artículos publicados en $P L O S$ según el indicador (comentarios, puntuaciones, bookmarks, enlaces blogs) presenten valores de 0 revela a las claras la escasa implicación de los investigadores en los servicios de la web social de la revista.

Esta situación puede explicarse por diversos factores. Por un lado, los aspectos técnicos juegan un papel no desdeñable. Para acceder a las funciones que miden la dimensión 1 de interacción y participación es necesario estar registrado en la revista, lo que ya supone una barrera de entrada. Igualmente para participar en la dimensión 3 de difusión 2.0 es necesario ser usuario de las aplicaciones (blogs y webs de etiquetado social). Por otra parte, la noción de recompensa es un factor determinante. Como señalan Neylon y Wu (2009), si no hay un sistema para recompensar los comentarios post-publicación en la comunidad científica no hay motivos para esforzarse realizando aportaciones que puedan añadir valor a los artículos. A ello se suma el hecho de que emitir opiniones, adelantar datos o realizar críticas plantea muchas interrogantes y ningún beneficio propio. Ofrecer pistas a competidores o enemistarse con posibles revisores y editores de revistas a los que podemos enviar nuestros trabajos son algunos de los riesgos que se corren en la ciencia abierta.

\section{"Indicadores como las citas o las descargas dan información útil tanto al editor como al investigador"}

Por último cabe señalar cómo ninguna pareja de indicadores presenta correlación significativa. Si bien para el caso de las descargas/ citas podemos explicar esta situación por medir dimensiones diferentes, para el resto de indicadores la explicación se debe a los escasos datos que son capaces de generar las funciones y aplicaciones 2.0 en relación con la publicación científica. En cualquier caso todos los indicadores nos ilustran los múltiples caminos y problemas a los que ya se enfrenta la medición y evaluación de la actividad científica.

\section{Bibliografía citada}

Cabezas-Clavijo, Álvaro; Torres-Salinas, Daniel; Delgado-López-Cózar, Emilio. "Ciencia 2.0: catálogo de herramientas e implicaciones para la actividad investigadora". El profesional de la informacion, 2009, v. 18, n. 1, pp. 72-79.

Evans, James A. "Electronic publication and the narrowing of science and scholarship". Science, 2008, v. 321, n. 5887, p. 395-399.

Kurtz, Michael J.; Bollen, Johan. "Usage biliometrics". Annual review of information science and technology, 2010, v. 44, pp. 3-64.

Neylon, Cameron; Wu, Shirley. "Article-level metrics and the evolution of scientific impact". PLoS biology, 2009, v. 7, n. 11, e1000242. http://www.plosbiology.org/article/info: doi/10.1371/journal.pbio.1000242

Torres-Salinas, Daniel; Delgado-López-Cozar, Emilio. "Estrategia para mejorar la difusión de los resultados de investigación con la web 2.0". El profesional de la información, 2009, v. 18, n. 5, pp. 534-539.

Álvaro Cabezas-Clavijo, EC3: Evaluación de la Ciencia y de la Comunicación Científica, Facultad de Comunicación y Documentación, Universidad de Granada. acabezasclavijo@gmail.com

Daniel Torres-Salinas, EC3: Evaluación de la Ciencia y de la Comunicación Científica. Centro de Investigación Médica Aplicada. Universidad de Navarra. torressalinas@gmail.com 